\title{
Datenanalytik am Lehrstuhl für Wirtschafts- und Betriebswissenschaften
}

\author{
Katharina Mertens ${ }^{1,2}$ \\ ${ }^{1}$ Leoben, Österreich \\ ${ }^{2}$ Lehrstuhl für Wirtschafts- und Betriebswissenschaften, Montanuniversität Leoben, Leoben, Österreich
}

Eingegangen 6. November 2018; angenommen 6. Dezember 2018; online publiziert 9. Januar 2019

Zusammenfassung: Die Mitarbeiter des Schwerpunkts Datenanalytik am Lehrstuhl für Wirtschafts- und Betriebswissenschaften der Montanuniversität Leoben setzen sich mit Daten von Unternehmen im Zuge von Projekten auseinander. Im Folgenden werden ein aktuelles Projekt sowie die Kompetenzen des Lehrstuhls vorgestellt.

\section{Schlüsselwörter: Datenanalytik, Datenanalytikprojekte}

Data Analytics at the Chair of Economic and Business Management

Abstract: The staff of the Chair of Economic and Business Management at the University of Leoben analyzes data provided by companies. In the following, a current project as well as the competences of the Chair are presented.

Keywords: Data analytics, Data analytics projects

\section{1. Überblick}

Die zunehmende Digitalisierung bringt einen Anstieg der Datenmenge, die zu entscheidungsrelevanten Informationen aufzubereiten ist, mit sich. Oftmals bestehen Problemstellungen, für die in einem ersten Schritt Daten generiert werden müssten. Die Ergebnisse der Datenanalytik ermöglichen die Optimierung von Prozessen und Produkten oder die Entwicklung neuer Geschäftsmodelle. Unabhängig von der Herangehensweise steht am Anfang eines Projekts mit datenanalytischem Schwerpunkt immer die Erfassung der Bedürfnisse und Anforderungen.

Durch die Ausbildung in unterschiedlichen Schwerpunkten besitzt das Datenanalytik-Team des Lehrstuhls für Wirtschafts- und Betriebswissenschaften genau diese Sichtweise. In der Optimierung von Instandhaltung und Produktion

DI K. Mertens, BSc $(\bowtie)$

Franz Josef-Straße 18,

8700 Leoben, Österreich

katharina.mertens@unileoben.ac.at

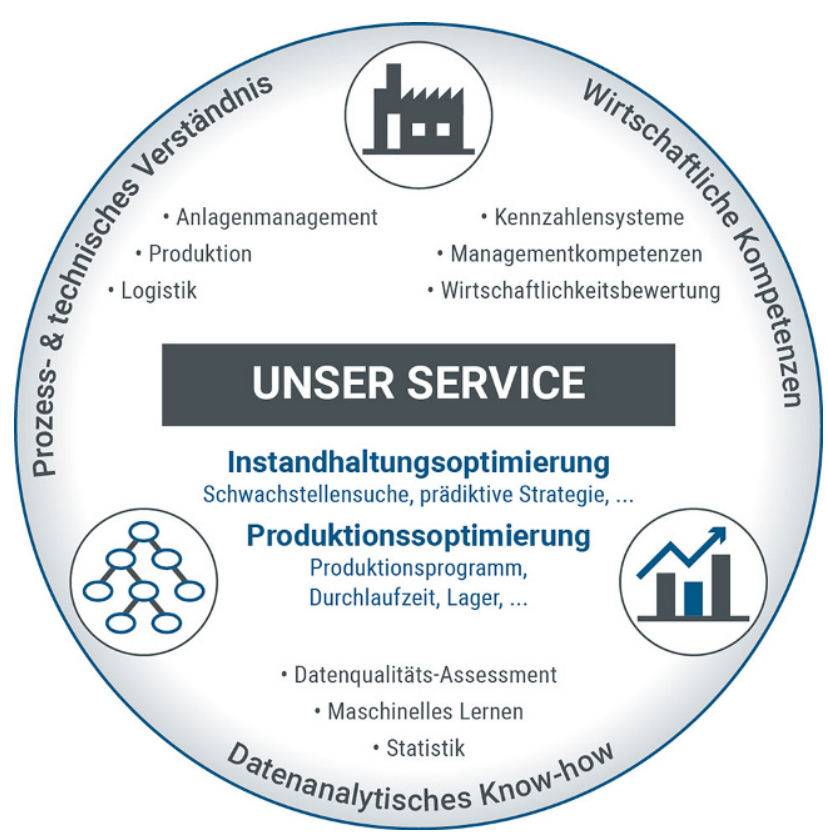

Abb. 1: Service des Lehrstuhls im Bereichs Datenanalytik

wird das Prozess- und Technikverständnis mit wirtschaftlichen Kompetenzen kombiniert. Dies ermöglicht es, maßgeschneiderte, wissenschaftlich fundierte Lösungen und Entscheidungshilfen zu entwickeln (Abb. 1).

\section{Kompetenzen}

Mit unterschiedlichen Hintergründen und Spezialisierungen deckt das Team verschiedene Bereiche und Tools ab, wozu seit neuestem das Process Mining zählt. Hierbei werden Prozesse aufgrund ihrer digitalen Spuren rekonstruiert. Dies erlaubt Prozesswissen, das sonst verborgen bleiben würde, zu visualisieren sowie greifbar und transportierbar zu machen. Einsatzmöglichkeiten können das Erfassen von 
Prozessen, für die keine formalen Beschreibungen vorhanden sind, oder der Vergleich von Soll- und Ist-Abläufen sein. Dies macht diese Technik auch für den Einsatz abseits reiner datengetriebenen Projekte attraktiv. Darüber hinaus hat das Datenanalytik-Team des Lehrstuhls folgenden Kompetenzen:

- Gezielte Forschungsaktivitäten im Bereich Produktions- und Anlagenmanagement mit Schwerpunkt Industrie 4.0 und Big Data Analytics

- Analysen in Richtung Energie-, Nachhaltigkeits-, Risikound Qualitätsmanagement

- Workshops für Unternehmen und Organisationen

- Einsatz spezifischer Tools (RapidMiner, Matlab, Python, Tableau)

- Strategie zur Datenerfassung in Industrie 4.0

- Machbarkeitsstudien

- Maßnahmenentwicklung - technisch, organisatorisch, personell

- Geschäftsmodellentwicklung

- Assessment zur Bestimmung der Reife von Daten

\section{Referenzprojekte}

Diese datengetriebene Projekte am Lehrstuhl für Wirtschafts- und Betriebswissenschaften gehen u. a. in Richtung Predictive Maintenance. Wann wird eine Maschine ausfallen? Welche Vorzeichen - Ursache-Wirkungsbeziehungen sind bekannt? Wie können dieses aufgezeigt werden?

Diese und eine Reihe anderer Fragen werden durch datenanalytische Methoden beantwortet. Hierbei dient der CRISP-DM (Cross Industry Standard Process for Data Mining) [1] als Rahmenwerk, an dem die Projektschritte ausgerichtet sind. Gemeinsam mit dem Projektpartner werden Voraussetzungen für den Einsatz von Datenanalytik geschaffen und Zusammenhänge erarbeitet oder aufgezeigt. Dies ermöglicht uns, maßgeschneiderte Lösungen und Entscheidungshilfen zu entwickeln.

Ein aktuelles Projekt ist die Anomalieerkennung an Altanlagen durch minimale Hardwarenachrüstung und Data Analytics [2]. Hierbei wurde in Zusammenarbeit mit einem externen Unternehmen eine Kältemaschine nachgerüstet und die dadurch erzeugten Daten analysiert. Ziel war es, Ausfälle vorherzusagen. Durch den hohen Wartungsgrad gab es keine Referenzwerte für Ausfälle. Daher wurde eine Anomalieerkennung durchgeführt - mit dem Hintergrund, dass eine Anomalie bzw. Abweichung vom Normalzustand mit der Anbahnung eines Ausfalls gleichzusetzen ist [2].

Funding. Open access funding provided by Montanuniversität Leoben.

Open Access Dieser Artikel wird unter der Creative Commons Namensnennung 4.0 International Lizenz (http://creativecommons.org/licenses/ by/4.0/deed.de) veröffentlicht, welche die Nutzung, Vervielfältigung, Bearbeitung, Verbreitung und Wiedergabe in jeglichem Medium und Format erlaubt, sofern Sie den/die ursprünglichen Autor(en) und die Quelle ordnungsgemäß nennen, einen Link zur Creative Commons Lizenz beifügen und angeben, ob Änderungen vorgenommen wurden.

\section{Literatur}

1. Shearer, C.: The CRISP-DM Model: The New Blueprint for Data Mining, Journal of Data Warehousing, 5 (2000), Nr. 4, S. 13-22

2. Bernerstätter, R.; Hirschmugl, R.: Anomalieerkennung an Altanlagen durch minimale Hardwarenachrüstung und Data Analytics, in: Biedermann, H. (Hrsg.): Predictive Maintenance: Realität und Vision. 32. Instandhaltungsforum der Österreichischen Vereinigung für Instandhaltung und Anlagenwirtschaft, 1. Aufl., Köln: TÜV Media, 2018, S. 199-212 